\title{
Stability of an erodible bed in various shear flows
}

\author{
Kouamé Kan Jacques KOUAKOU ${ }^{1,2}$ \\ Pierre-Yves LAGRÉE ${ }^{2}$ \\ 1- Laboratoire de Mécanique, Université de Cocody, \\ Abidjan, Côte d'Ivoire \\ 2- Laboratoire de Modélisation en Mécanique, U.M.R. CNRS 7607, \\ Université Pierre et Marie Curie, Boîte 162, 4 place Jussieu, \\ 75252 Paris Cedex 05, France. \\ mess.e.: pyl@ccr.jussieu.fr
}

October 5, 2004

\begin{abstract}
The 2D laminar quasi-steady asymptotically simplified and linearized flow with mass transport of sediments is solved over an erodible bed in various laminar basic shear flow (steady, oscillating or decelerating). The simplified mass transport equation includes the two following phenomena: flux of erosion when the skin friction goes over a threshold value, and a non local effect coming either from an inertial effect or from a slope effect. It is shown that the bed is always unstable for small wave numbers. Examples of long time evolution in various shear régimes are presented, wave trains of ripples are created and merge into a unique bump. This coarsening process is such that the maximum wave length obeys a power law with time. 45.70.-n Granular systems 47.15.Cb Laminar boundary layers 45.70.Qj Pattern formation
\end{abstract}

\section{Introduction}

Understanding how water or air creates ripples on sand or sediments is a very important environmental problem. Widely different wave lengths are involved from small ripples on the beach (either in the wet or dry sand) to dune formation or mega ripples in sea. A large literature refers to this problem since Exner in 1925 and Bagnold in the 40's up to now, as ripples on Mars may be an evidence of water on this planet [6]. Yang [28], Fredsøe and R. Deigaard [7], and Nielsen [15] give the state of the art for this very complex problem linking various physical aspects at different scales (from the grain diameter to the sea depth!).

Experimental setup have been constructed to study how ripples appear and grow. Typical experimental setups are oscillating annular cells (Sherer et al. [20], Rousseaux et al. [17]), uniformly rotating cell (Charru et al. [5]) or stopping a uniformly rotating cell (Caps [4]). It is observed that as time increases the wave length of the ripple increases (coarsening of the ripples).

We simulate this phenomena with a simple model for both the flow and the soil. Instead of using an amplitude model equation we want to 
use model equations coming from mechanics. The first difficulty consists in computing the flow which is unsteady and turbulent in the nature, but which may be considered as laminar in most experiments. Our model will consider quasi steady laminar perturbations of a slowly varying basic flow. An asymptotical approach allows to obtain a linearised solution for the flow over the ripples. The second difficulty consists in computing the flow of sand. The sand may "creep" (bed load transport), or "fly", or may be suspended in the fluid. This modifies the viscosity and density of the flow. Our model will consider different simple relations between the flux of sand and the shear stress from the flow.

We present a stability theory for different cases of flow. The basic state is a flat erodible bed with a shear flow. Either a steady established flow, an oscillating one, or a decelerated one will be considered. The questions are about the stability of the configuration, and about the long time evolution of the bed form.

We first remind the equations of the full coupled problem (Sec. 2) and the first simplifications for the flow and for the granular material. We present the simple basic régimes, they reduce near the soil to a shear flow (Sec. 3). We focus on the small perturbations of this flow in section 4 . The linear stability is then discussed (Sec. 5). Finally we present a long time evolution (Sec. 6) of the erodible bed leading to the coarsening of wave length.

\section{The coupled problem}

\subsection{The flow}

We consider a steady or unsteady laminar incompressible Newtonian flow. We suppose that the viscosity and density remain constant in the flow. An initial boundary layer is developing, $\delta$ is its thickness, $U_{0}$ is the velocity far from the soil. The Reynolds number $R e_{\delta}=\frac{U_{0} \delta}{\nu}$ is supposed large enough to use an asymptotical approach. In fact the slope of the velocity at the surface $U_{0} / \delta$ will be the pertinent parameter. We will specialise the boundary layer in section (3). Knowing the instantaneous soil shape the problem is to find the velocity near the soil, more exactly the skin friction $\tau=\mu \partial_{y} u$ from the Navier Stokes equations. Using $\delta$ as length scale, $y=\delta \bar{y}, x=\delta \bar{x}, u=U_{0} \bar{u}$, and $t=\left(\delta^{2} / \nu\right) \bar{t}$. The Navier Stokes equations are (Rousseaux et al. [18]):

$$
\begin{array}{r}
\frac{\partial \bar{u}}{\partial \bar{x}}+\frac{\partial \bar{v}}{\partial \bar{y}}=0, \\
\frac{1}{\operatorname{Re} \delta} \frac{\partial(\bar{u}, \bar{v})}{\partial \bar{t}}+(\bar{u}, \bar{v}) \cdot \bar{\nabla}(\bar{u}, \bar{v})=-\bar{\nabla} \bar{p}+\frac{1}{\operatorname{Re}_{\delta}} \bar{\nabla}^{2}(\bar{u}, \bar{v}) .
\end{array}
$$

Here we will concentrate to the case of small perturbations of the soil's surface, and we will see that asymptotic analysis allows an analytical resolution. The boundary conditions are the no slip flow at the upper surface of the soil described by $\bar{y}=\bar{f}$.

\subsection{The erodible bed}

Due to the movement of sand or sediment, the upper surface of the soil $(y=f(x, t))$ changes according to the mass conservation. To solve the 
mass conservation of sediments

$$
\frac{\partial f}{\partial t}=-\frac{\partial q}{\partial x}
$$

one has to know the relation between the flux $q$ and the fluid flow. This depends on a lot of factors. In the literature (Yang [28], Nielsen [15], Fredsøe and Deigaard [7], ...) the final relation is mostly that $q$ is function of the skin friction $\tau$. The adimensionalised skin friction is denoted as the Shield number $\theta=\frac{\tau}{\left(\rho_{s}-\rho\right) g d} ; \rho$ and $\rho_{s}$ are fluid and particle density, respectively, $d$ is average particle diameter, and $g$ is gravity. There is a threshold value $\tau_{s}\left(\right.$ or $\left.\theta_{s}\right)$ above which the sediments are entrained, a power law (with often $\beta=3 / 2$ ) is often used:

$$
q_{s} \propto\left(\theta-\theta_{s}\right)^{\beta}, \text { with } \theta>\theta_{s} .
$$

In fact, we use a linearized version just above the threshold: $q_{s} \propto \tau-\tau_{s}$. Furthermore, there is a gravity effect which induces a correction in the threshold value depending on the slope of the soil. If $\tau>\tau_{s}+\Lambda \frac{\partial f}{\partial x}$ we define a saturated flux:

$$
q_{s}=Q_{0} \varpi\left(\tau-\tau_{s}-\Lambda \frac{\partial f}{\partial x}\right)
$$

where $\varpi(x)=x$ if $x>0$, else $\varpi(x)=0$. In an eolian context, Kroy et al. [11], Sauermann et al. [19] introduced an effect of inertia adding a term proportional to $\partial_{x} q$ (with $\Lambda=0$ ), we may simplify their expression as:

$$
l_{K} \frac{\partial q}{\partial x}+q=q_{s}
$$

with $l_{K}$ proportional to $\frac{1}{U_{S}^{\prime}}$ i.e. the inverse of the shear. With again $\Lambda=0$, Andreotti et al. [1] simplified this expression as:

$$
l_{s} \frac{\partial q}{\partial x}+q=q_{s}
$$

with a constant coefficient in front of $\partial_{x} q$. Finally the slope of the ripple may be limited by an "avalanche" effect (at least a "slope effect"). It means that at each time step:

$$
\text { if }\left|\frac{\partial f}{\partial x}\right|>\frac{1}{\mu} \text {, then }\left|\frac{\partial f}{\partial x}\right|=\frac{1}{\mu} \quad \text { and } \quad \int f d x=\text { cste. }
$$

Mass is conserved during this process, and slope is limited. The problem is now to find $\tau$ and to test either Eq. 4, 5, or 6, and 7 .

\section{Basic flow}

The basic configuration is a bidimensional flow over a flat soil. Curvature and gap effects in circular cells are neglected. The flow is laminar. Near the wall, any velocity profile reduces to a pure shear flow (Figure 1). The basic adimensionalised velocity is:

$$
\bar{u}=\bar{U}_{S}^{\prime} \bar{y} .
$$

Typical examples are an oscillating flow, or a moving tank with an impulsively stopped bottom or a Blasius boundary layer. In the next subsections we will show that the steady case corresponds to $\bar{U}_{S}^{\prime}=1$, the oscillating case corresponds to $\bar{U}_{S}^{\prime}=\cos (2 \pi \bar{t})$, and the decelerated case to $\bar{U}_{S}^{\prime}=\bar{t}^{-1 / 2}$. 


\subsection{Steady basic flow}

If we consider a Blasius boundary layer, near the wall, the profile is linear (Schlichting [24]):

$$
u \simeq 0.33 U_{0} \frac{y}{\delta}
$$

with $\delta=\left(L R_{L}^{-1 / 2}\right)$ so that we obtain (8) with $\bar{U}_{S}^{\prime}=0.33$. We may as well imagine a flow in a channel with a developed half Poiseuille profile, the parabolic velocity profile being linear near the wall. The analysis of Fowler [8] of a turbulent flow with a mean equivalent viscosity leads in fact to this analysis.

\subsection{Oscillating basic flow}

In the frame of the wall, the basic flow is solution of:

$$
\frac{\partial}{\partial t} u=-\frac{d}{\rho d x} p+\nu \frac{\partial^{2}}{\partial y^{2}} u \text {. }
$$

The oscillating velocity $U_{0} \Re[\exp (-i \Omega t)]$ is imposed at "infinity", far from the bottom where $u(y=0, t)=0$. The solution is classical (Schlichting [24]) and after a change of phase in the time, near the wall:

$$
u \simeq U_{0} \frac{y}{\delta} e^{-i \Omega t}+\ldots
$$

so that we obtain (8) with $\bar{U}_{S}^{\prime}=\cos (2 \pi \bar{t})$, with time scaled with $f^{-1}=$ $2 \pi \Omega^{-1}$. The scale of the boundary layer $\delta=(\nu / \Omega)^{1 / 2}$.

\subsection{Decelerated Basic flow}

In the frame of the wall, the basic flow is again the solution of Eq. (10) (Rayleigh problem) with $U_{0}$ the constant velocity at "infinity", far from the bottom and $u(y=0, t)=0$ :

$$
u \simeq U_{0} \frac{y}{\sqrt{\pi \nu t}}+\ldots
$$

so that we obtain (8) with $\bar{U}_{S}^{\prime}=1 / \sqrt{\bar{t}}, \delta=\left(\pi \nu T^{*}\right)^{1 / 2}$ were $T^{*}=h_{0}^{2} / \nu$ is the chosen time scale $\left(h_{0}\right.$ is the water depth, which is supposed larger than $\delta$ ).

\section{Perturbation of the basic flow}

In a layer of relative thickness $\varepsilon$ (compared to the uniform boundary layer thickness $\delta$ ), and longitudinaly streched of a factor $\lambda / \delta$ we may develop (12) using $\tilde{y}=\varepsilon^{-1} \bar{y}=\frac{y}{\varepsilon \delta}$ and $\tilde{x}=\frac{\delta}{\lambda} \bar{x}=\frac{x}{\lambda}$. Perturbations of velocity must be of order $\varepsilon$, in order to reobtain far upstream of a bump: $\tilde{u}=\bar{U}_{S}^{\prime} \tilde{y}$. To have a problem with the maximum number of terms (least possible degeneracy, Van Dyke [26]), including pressure, convective terms and a viscous term, we obtain a relation between $\lambda$ and $\delta$ :

$$
\lambda=\varepsilon^{3} \delta R_{\delta}
$$


So with $\bar{x}=\tilde{x}, \bar{y}=\varepsilon \tilde{y}, \bar{u}=\varepsilon \tilde{u}, \bar{v}=\varepsilon \tilde{v}$, and $\bar{p}=\varepsilon^{2} \tilde{p}$, and if we take the time scale to be the time scale of the oscillating flow $\bar{t}=\tilde{t}$. This means that we have:

$$
\varepsilon^{2} \frac{\partial}{\partial \tilde{t}} \tilde{u}+\tilde{u} \frac{\partial}{\partial \tilde{x}} \tilde{u}+\tilde{v} \frac{\partial}{\partial \tilde{y}} \tilde{u}=-\frac{\partial}{\partial \tilde{x}} \tilde{p}+\frac{\partial^{2}}{\partial \tilde{y}^{2}} \tilde{u}+\varepsilon^{2} \frac{\partial^{2}}{\partial \tilde{x}^{2}} \tilde{u} .
$$

and $-\frac{\partial}{\partial \tilde{y}} \tilde{p}=O\left(\varepsilon^{2}\right)$. So that, as $\varepsilon \rightarrow 0$, the problem is quasisteady, and we obtain a "boundary layer" equation for $\tilde{u}$ (Eq. 15).

\subsection{Notes on the scalings}

We note $($ as $\varepsilon \rightarrow 0)$ that the second order partial longitudinal derivative is not present. It is because the transverse scale is smaller than the longitudinal one. Using the same scale in both directions $x=\lambda_{v} \hat{x}$ and $y=\lambda_{v} \hat{y}$, we have $\lambda_{v}=\sqrt{\nu /\left(U_{0} / \delta\right)}$ and Eq 2, for $\hat{u}$ is:

$$
\varepsilon^{2} \frac{\partial \hat{u}}{\partial \hat{t}}+\hat{u} \frac{\partial \hat{u}}{\partial \hat{x}}+\hat{v} \frac{\partial \hat{u}}{\partial \hat{y}}=-\frac{\partial}{\partial \hat{x}} \hat{p}+\frac{\partial^{2}}{\partial \hat{y}^{2}} \hat{u}+\frac{\partial^{2}}{\partial \hat{x}^{2}} \hat{u} .
$$

This scale is called the "viscous scale" (Charru et al. [5]), it is supposed very small. In fact we are in the Triple Deck theory (Neiland [14], Stewartson \& Williams [23], Smith [21], Sychev et al [25]), and Gajjar \& Smith [9], Bowles \& Smith [2]). More precisely, we are in the framework of Double Deck Smith (1982) [21]. We focus on the case with no perturbations of the Upper Deck (no retroaction of the ideal fluid). This means that perturbations of pressure induced by the deflection of the streamlines in the Upper Deck (of order $\frac{\varepsilon \delta}{\lambda \varepsilon^{3} \delta}$ ) are smaller than the perturbation of pressure in the Lower Deck (of order $\varepsilon^{2}$ ) (Smith et al. [22]). In the Blasius case, this means that $\varepsilon<<R_{L}^{-1 / 8}$.

In complete Triple Deck, time should then be scaled by $\varepsilon^{2}$ compared to the time scale of the basic flow in this lower layer. But this is not relevant here because this introduces a smaller time scale than the one coming from the flow (i.e. $1 / \Omega$ in the oscillating case). Furthermore, the time of variation of the soil is itself very long compared to the oscillation period.

\subsection{Equations}

Steady and unsteady cases follow the same equations, the problem in the "Lower Deck" is simply:

$$
\begin{aligned}
\frac{\partial}{\partial \tilde{x}} \tilde{u}+\frac{\partial}{\partial \tilde{y}} \tilde{v} & =0, \\
\tilde{u} \frac{\partial}{\partial \tilde{x}} \tilde{u}+\tilde{v} \frac{\partial}{\partial \tilde{y}} \tilde{u} & =-\frac{d}{d \tilde{x}} \tilde{p}+\frac{\partial^{2}}{\partial \tilde{y}^{2}} \tilde{u}
\end{aligned}
$$

It means that near the wall there exist scales such that a non linear problem (with convection, diffusion and pressure gradient) has to be solved. Boundary conditions are no slip condition on the bottom:

$$
\tilde{u}(\tilde{x}, \tilde{y}=\tilde{f}(\tilde{x}))=0, \quad \tilde{v}(\tilde{x}, \tilde{y}=\tilde{f}(\tilde{x}))=0 .
$$

The matching between the top of the Lower Deck $(\tilde{y} \rightarrow \infty)$ and the bottom of the boundary layer $(\bar{y} \rightarrow 0)$ gives:

$$
\lim _{\tilde{y} \rightarrow \infty}(\varepsilon \tilde{u}(\tilde{x}, \tilde{y}))=\bar{U}_{S}^{\prime} \bar{y} \quad \text { i.e. } \quad \tilde{u}(\tilde{x}, \tilde{y} \rightarrow \infty)=\bar{U}_{S}^{\prime} \tilde{y}
$$


The latter means that the incoming velocity is linear, this means that upstream we recover the boundary layer profile:

$$
\tilde{u}(\tilde{x} \rightarrow-\infty, \tilde{y})=\bar{U}_{S}^{\prime} \tilde{y}, \quad \tilde{v}(\tilde{x} \rightarrow-\infty, \tilde{y})=0 .
$$

\subsection{Linearisation: law between the topography and the skin friction.}

The unperturbed solution of (14-15) is simply:

$$
\tilde{u}=\bar{U}_{S}^{\prime} \tilde{y}, \quad \tilde{v}=0, \quad \tilde{p}=0 .
$$

This means that at the small longitudinal scale, the boundary layer thickness does not evolve and the velocity profile remains linear near the wall. The linearised solution of (14-15) around this shear profile in Fourier space is straightforward and leads to ( $A i$ is the Airy function):

$$
\tilde{\tau}=\bar{U}_{S}^{\prime}+\bar{U}_{S}^{\prime} F T^{-1}\left[F T[\tilde{f}](3 A i(0))\left(-(i \tilde{k}) \bar{U}_{S}^{\prime}\right)^{1 / 3}\right]+\ldots
$$

This relation (19) gives the final response of the fluid: it links the perturbation of the shear stress to the topography change. This final relation will be used for the linear stability analysis as well as for the numerical simulations.

\section{Linear stability of the bed}

\section{1 steady case}

The shear stress function of the topography change is now coupled to the adimensional version of (4),

$$
\tilde{q}_{s}=\varpi\left(\tilde{\tau}-\tilde{\tau}_{s}-\tilde{\Lambda} \frac{\partial \tilde{f}}{\partial \tilde{x}}\right) .
$$

Either the flux is always saturated and

$$
\tilde{q}=\tilde{q}_{s},
$$

or the inertial effect is taken into account with a variable coefficient (5)

$$
\frac{\tilde{l}_{K}}{\bar{U}_{S}^{\prime}} \frac{\partial \tilde{q}}{\partial \tilde{x}}+\tilde{q}=\tilde{q}_{s}
$$

or the inertial effect is taken into account with a constant coefficient (6)

$$
\tilde{l}_{s} \frac{\partial \tilde{q}}{\partial \tilde{x}}+\tilde{q}=\tilde{q}_{s}
$$

where $\tilde{\tau}_{s}, \tilde{\Lambda}, \tilde{l}_{K}$, and $\tilde{l}_{s}$ are constants. In practice, in the sequel, we do not mix the effect of inertia $\frac{\partial \tilde{q}}{\partial \tilde{x}}$ and the avalanche (or slope) effect $\frac{\partial \tilde{f}}{\partial \tilde{x}}$, so in (22 and 23) $\Lambda$ is zero in $\tilde{q}_{s}$. The bed form evolution (3) with a suitable adimensionalisation is:

$$
\frac{\partial \tilde{f}}{\partial \bar{t}}=-\eta \frac{\partial \tilde{q}}{\partial \tilde{x}}
$$

where $\eta$ represents the ratio of the hydrodynamic scale versus the bed evolution one. In pratice this arises only when $\bar{U}_{S}^{\prime}$ is not constant. When $\bar{U}_{S}^{\prime}$ is constant, the time scale is such that $\eta=1$. We introduce a long 
time $\bar{t}_{1}=\eta \bar{t}$. This allows to compute the temporal stability of an erodible bed with $\bar{U}_{S}^{\prime}$ given. Looking for normal modes $\tilde{f}=e^{(\sigma+i \omega) \bar{t}_{1}} e^{-i k \tilde{x}}$, we obtain the perturbation of the skin friction (Eq. 19) and with the suitably adimensionalised version of flux relation (Eq. 21 or 23 or 22), we obtain $\sigma$ as a function of the mode $k$. We note that there exist a uniform flux:

$$
\tilde{q}_{0}=\bar{U}_{S}^{\prime}-\tilde{\tau}_{s} .
$$

With $\tilde{q}_{0}>0$, the linear stability analysis is valid as long $\tilde{\tau}-\tilde{\tau}_{s}>0$. The amplification factor $\sigma$ is positive for small $k$ (with Eq. 4):

$$
\sigma+i \omega=\frac{3^{\frac{1}{3}}}{\Gamma\left(\frac{2}{3}\right)}\left(\frac{1}{2}+\frac{i \sqrt{3}}{2}\right) k^{4 / 3}-\tilde{\Lambda} k^{2}
$$

Unstability occurs only for long waves (figure 2). If the slope effect is removed $\left(\tilde{\Lambda}=0\right.$ in Eq. 4), all waves are amplified $\left(\sigma=0.53 k^{4 / 3}\right.$ and $\omega=0.92 k^{4 / 3}$ ). The same arises for the case with saturation effect (Eq. 6 or 5 here identical), there is unstability only for long waves (figure 3 ).

Figure 4 gives an interpretation of the unstability, a wavy profile has an excess of skin friction $\tilde{\tau}-\bar{U}_{S}^{\prime}$ that is maximal just before the crest. The flux is decomposed in a uniform flux $\tilde{q}_{0}$ plus a perturbation which is $\tilde{\tau}-\bar{U}_{S}^{\prime}$. This latter contribution erodes the crest and displaces the matter down stream.

\subsection{Oscillating case}

\subsubsection{Averaging the flux relation}

The oscillating time scale is supposed smaller than the time of growth of the structures. We have again (24) with $\eta$ ratio of the oscillating time by the bed evolution one. We define a mean value $\langle\cdot\rangle=\int_{0}^{1} \cdot d \bar{t}$ during an oscillating cycle. Introducing a multiscale analysis: $\bar{t}_{0}=\bar{t}$ is the short time, and $\bar{t}_{1}=\eta \bar{t}$ is the long time. Let split $\tilde{f}=\tilde{f}_{0}\left(\bar{t}_{0}, \bar{t}_{1}\right)+\eta \tilde{f}_{1}\left(\bar{t}_{0}, \bar{t}_{1}\right)+\ldots$ and write $\tilde{q}=\tilde{Q}+\tilde{q}^{\prime}$. $\tilde{Q}$ is defined as the mean value during the current cycle $\tilde{Q}=<\tilde{q}>$ and $<\tilde{q}^{\prime}>=0$. So, as the time derivative is: $\frac{\partial}{\partial t}=$ $\frac{\partial}{\partial t_{0}}+\eta \frac{\partial}{\partial t_{1}}$, the mass conservation equation degenerates:

$$
\frac{\partial \tilde{f}_{0}}{\partial \bar{t}_{0}}=0
$$

i.e. the topology is quasisteady at the short time scale. We define $\tilde{F}_{0}\left(\bar{t}_{1}\right)=$ $\tilde{f}_{0}\left(\bar{t}_{0}, \bar{t}_{1}\right)$. At the long time scale:

$$
\frac{\partial \tilde{F}_{0}}{\partial \bar{t}_{1}}+\frac{\partial \tilde{f}_{1}}{\partial \bar{t}_{0}}=-\frac{\partial \tilde{q}}{\partial \tilde{x}} .
$$

With the decomposition of $q$ :

$$
\frac{\partial \tilde{f}_{1}}{\partial \bar{t}_{0}}=\left(-\frac{\partial \tilde{q}^{\prime}}{\partial \tilde{x}}\right)+\left(-\frac{\partial \tilde{Q}}{\partial \tilde{x}}-\frac{\partial \tilde{F}_{0}}{\partial \bar{t}_{1}}\right) .
$$

In order to solve the problem at order one, the secular term: $\left(-\frac{\partial \tilde{Q}}{\partial \tilde{x}}-\frac{\partial \tilde{F}_{0}}{\partial t_{1}}\right)$ must be 0 :

$$
\frac{\partial \tilde{F}_{0}}{\partial \bar{t}_{1}}=-\frac{\partial<\tilde{q}>}{\partial \tilde{x}}
$$

This means that we can take the mean value of $q$ to deal with the long time evolution of the bed. 


\subsection{2 $\quad$ stability}

During a cycle the ripples do not change at first order, the time of evolution of the topography is very slow compared to the oscillating time. The small short time pertubation perturbation of the soil is given by $\partial_{\bar{t}_{0}} \tilde{f}_{1}=-\partial_{\tilde{x}} \tilde{q}^{\prime}$, where $\tilde{q}^{\prime}$ must be borned. So we estimate the mean value of the skin friction with a "frozen" soil from Eq. 19 during a cycle (note $<\bar{U}_{S}^{\prime}>=0$, and we use $\tilde{f}$ which is in fact $\left.\tilde{F}_{0}\right)$ :

$$
<F T[\tilde{\tau}]>=-3 A i(0) \frac{i \Gamma\left(\frac{1}{6}\right)}{8 \sqrt{\pi} \Gamma\left(\frac{2}{3}\right)}(k)^{1 / 3} F T[\tilde{f}]
$$

$<\tilde{\tau}>$ and $\tilde{f}$ are out of phase, the skin friction will induce the sand to move to the crests and out of the hollow (figure 7 ) without displacing the ripples. The mean value of the skin friction is real:

$$
\int_{0}^{1}-(-i k) F T[\tau] d \bar{t}=\frac{3^{\frac{1}{3}} \Gamma\left(\frac{1}{6}\right) k^{4 / 3} T F[\tilde{f}]}{8 \sqrt{\pi} \Gamma\left(\frac{2}{3}\right)^{2}},
$$

the numerical value is $0.3087 T F[\tilde{f}]$.

Putting the slope effect (21), the stability analysis gives:

$$
\sigma=0.3087 k^{4 / 3}-\Lambda k^{2}, \quad \omega=0 .
$$

There is a cut off frequency $k_{c}$. Stability for large $k: k>k_{c}$ and instability for $k<k_{c}$. See fig 5 . Taking a relation with an inertial effect depending on the actual value of the shear stress (22) with $\tilde{q}_{s}=\tau$ gives:

$$
\sigma+i \omega=<-(-i k) \frac{\tilde{U}_{S}^{\prime} 3 A i(0)\left(-(i k) \bar{U}_{S}^{\prime}\right)^{1 / 3}}{\left.1+\tilde{l}_{K}\left(i k\left(\bar{U}_{S}^{\prime}\right)^{-1}\right)\right)}>,
$$

which is real $(\omega=0)$, and plotted on fig. 6. As for the steady case, long waves are unstable, in this oscillating case there is no phase velocity. The ripples do not move.

\subsection{Decelerated case}

In the previous section, the hydrodynamic time was smaller than the bed time evolution. If we look at a decelerated flow, generally no ripples are created. The time during which the flow exists is too short for ripples to grow. In some cases, this arises (Caps [4]). In order to describe this case, the time scale of the flow must be the same than the time scale of the bed. Taking the same scale for the flow and the topology variation we have (in the case (21)):

$$
\frac{\partial \tilde{f}_{k}}{\partial \bar{t}}=\left(-3 A i(0)(-i k)(-i k)^{1 / 3} \bar{t}^{-2 / 3}-\Lambda k^{2}\right) \tilde{f}_{k}
$$

Which may be solved for each Fourier mode $k$ :

$$
\log \left(\tilde{f}_{k}\right)=-9 A i(0)(-i k)(-i k)^{1 / 3} \bar{t}^{-1 / 3}-\Lambda k^{2} \bar{t} .
$$

Stability analysis now deals with $\log \left(\tilde{f}_{k}\right)$. On figure 8 is displayed $\log \left(\tilde{f}_{k}\right)$ as function of $k$ for various time. As time increases, the cut off frequency $k_{c}$ decreases. Short waves are more and more stabilised. 


\subsection{Conclusion for stability analysis}

As a conclusion for this section devoted to linear stability analysis, we observe that the qualitative behaviour is the same in every case: the long wave are unstable, the short wave are stabilised by the gravity or the inertia effect. In the oscillating case, there is no downstream propagation of the ripples.

\section{Examples of large time evolution}

\section{1 numerical method}

The system is solved numerically using a fast Fourier transform ([16]). The non linearity of the problem is taken in real space and consists in the fonction $\varpi$ that is zero under the threshold. The initial profile is a random small noise. We observe (see following figures) that the time for ripples to grow and to interact is very long. Therefore we solved the oscillating case with $\eta=1$ (taking at each time step $\bar{t}$ the exact value of $\tilde{\tau}$ ). This is a simple way to tackle with the problem of the non linearity induced by $\varpi$. We obtain a coarsening in all the configurations.

\subsection{Steady case}

Starting from a random noise, the structures predicted by the linear theory appear. As time increases, the non linear stage is induced by the threshold function $\varpi$. The sinusoidal shape is transformed in a non symmetrical shape (see fig. 9 a spatio temporal diagram). The ripples move from left to right. The ripple downstream of a larger one is eaten by it. This is due to the fact that the lee bumps experiment a smaller skin friction than the one upstsream. As a result, there is less and less bumps in the computational domain: this is "coarsening". Finally there is only one bump in the "box". On figure 10 is plotted the number of bumps which diminishes whith time, whereas the bump height increases with time. The eight of the final bump saturates. The wave length $\lambda_{\max }$ (which corresponds to the distance between the bumps, and is inversely proportional to the number of bumps) increases. On fig. 11 is plotted this wave length for several simulation with various values of domain size, values of threshold $\tau_{s}$ and models (either 21 or 22). The large time behaviour is the same, this is due to the fact that at small wave length $k$ the bump is large so the exact value of threshod does not matter anymore. Furthermore, as the wave length is small, Eq. 21 or Eq. 22 are identical and reduce to

$$
\tilde{q}=\tilde{\tau}+\ldots
$$

The wave length $\lambda_{\max }$ is plotted as function of $t$ in fig 11. A log-log plot suggest that $\lambda_{\max }$ is more or less proportional to time, it seems that: $\lambda_{\max } \propto \bar{t}$.

\subsection{Oscillating case}

Starting again from a random noise, the structures predicted by the linear theory appear. As time increases, the non linear stage is induced by the threshold function $\varpi$. The sinusoidal shape is transformed in a symmetrical shape (right/ left, see fig. 13) due to the symmetry of the flow. The ripples are steady and only move from one to the other during the 
pairing process. Again, there is "coarsening": less and less bumps are in the computational domain. Finally there is only one bump in the "box". On figure 12 the same is observed in the case of slope limitation (7). On figure 14 is plotted the number of bumps which diminishes, whereas the bump height increases. The wave length $\lambda_{\max }$ increases. On fig. 15 is plotted this wave length for several simulations with various values of domain size, values of threshold $\tau_{s}$ and models (either 21 or 22). The large time behaviour is again the same. The wave length $\lambda_{\max }$ is plotted as function of $t$ in fig 15. A log-log plot suggest that is near a $2 / 3$ power: $\lambda_{\max } \propto t^{0.6}$ which is different from the steady case (fig 11). Experimentaly Rousseaux et al. [17] studied ripples from the rolling ripple stage to the vortex ripple one. The amplitude of the movement is $A$ (typically $1 \mathrm{~cm}$ ) and the frequency is $f$ (typically $1 \mathrm{~Hz})$ ), so that $U_{0}=2 A \pi f$ and $\delta=\sqrt{(\nu /(\pi f)}$. Then our theory apply for any $\varepsilon$ such that Eq. 13 gives: $\lambda=\varepsilon^{3}(2 A)$. Our theory is then valid as long as the wave length are smaller than the amplitude of the movement. It seems to be nearly the case in this experimental setup where $0.01 m<A<0.05 m$ and where the first measured wave length is $\lambda_{\text {initial }} \simeq 0.005 \mathrm{~m}$. Furthermore, even if our theory does not strictly compute the separation, the prediction of formula 19 is a good one even when there is flow separation (Lagrée [13]). Our theory is a good approximation of the flow with small separation bubbles. So we model the rolling ripples and the vortex ripple when they are small. The Cahn-Hillard models (Villain-Guillot, and Josserand [27], Bray [3]) predict that $\lambda_{\max } \propto \log (t)$. Rousseaux et al. [17] suggest (but maybe on a too small range of time) this fit for their data. With our model, on a larger range, we instead have a power law.

\subsection{Decelerated case}

As already mentioned, the time to obtain the growth of ripples is long, here the time is borned (there exist a time at which $\bar{U}_{S}^{\prime}=\tilde{\tau}_{s}$ ). So $\tilde{\tau}_{s}$ must be enough small. The final time of growth is a bit larger than the time at which $\bar{U}_{S}^{\prime}=\tilde{\tau}_{s}$. On figure 16 is a spatio-temporal diagram. As the effect of a bump is to increase the skin friction, there is an excess of $\tilde{\tau}-\tilde{\tau}_{s}$ that is only due to the crest of the ripple.

\section{Conclusion}

A model for the growth of ripples in a laminar 2D shear flow has been presented. A condition for application is that the height of the formed ripples must be smaller than the boundary layer thickness of the basic flow in order to obtain a basic shear flow.

This shear flow may be steady or slowly varying in time (oscillating or decelerating). A linear solution linking the bed shape and the skin friction is obtained in Fourier space. Depending on the chosen relation linking the transport of sediments and the shear (or skin friction) we obtain a coupled problem. We present a stability analysis of the erodible bed. In the chosen framework, in every case, the bed is unstable for long waves. The instability is due to the fact that the skin friction is in advance of phase with the soil. The short wave length are stabilised by either an "inertial" effect, either an "avalanche" effect. In the oscillating case, during a period, the bed does not change that allows a multiscale analysis of the instability. The stabilising effect for the short waves is the 
same. In the decelerated case, the classical temporal stability analysis has to be changed because the time of the deceleration and the time of growth of the structures must be the same.

Large time simulation of the model equations shows that the ripples merge and we obtain only one bump in the domain (except in the decelerated case because shear is decreasing finally under the threshold). During the coarsening process, the history is independent of the exact value of the parameters. The maximal wave length $\lambda_{\max }$ is proportional to a power of time. 


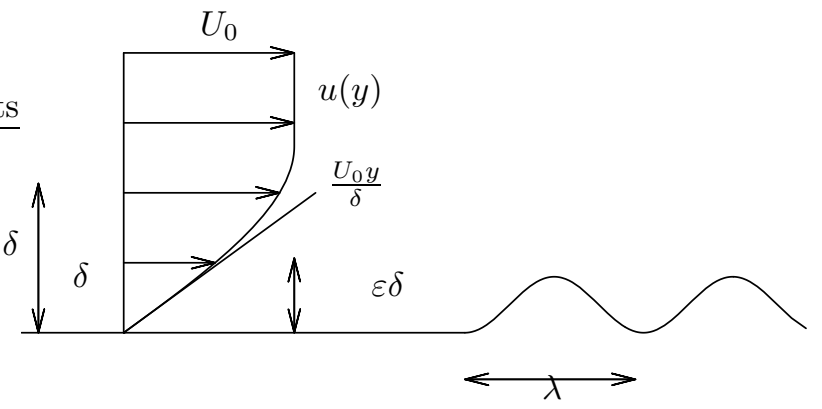

Figure 1: Any velocity profile is linear near the wall. $\lambda$ is a typical length.

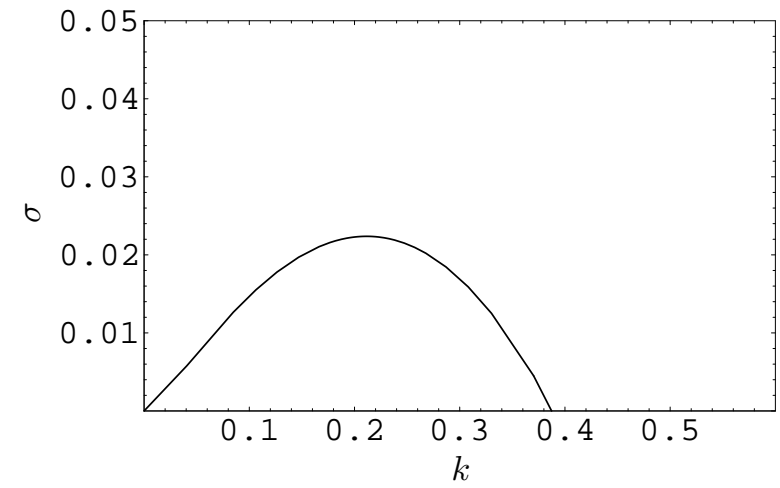

Figure 2: Constant shear, $\bar{U}_{S}^{\prime}=1$, amplification factor $\sigma$ as function of number $k$, case ( 4 or 21 , and 25$)$ with $\tilde{\Lambda}=1$, decreasing $\tilde{\Lambda}$ increases the cut off value of $k$. 


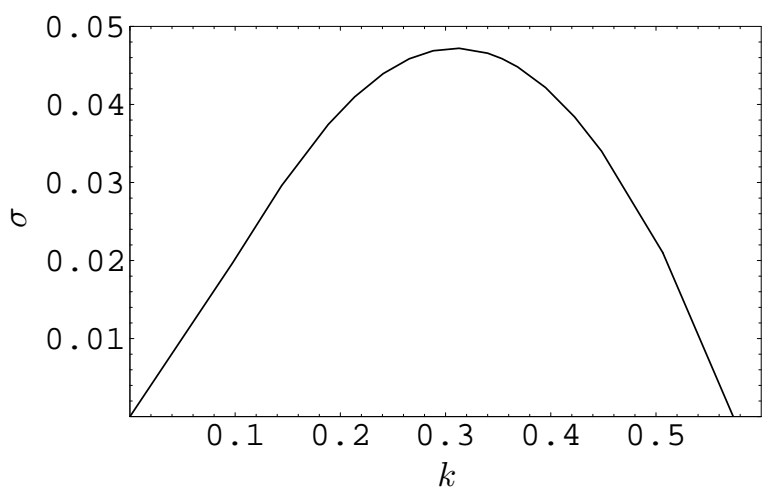

Figure 3: Constant shear, $\bar{U}_{S}^{\prime}=1$, amplification factor $\sigma$ as function of number $k$, case (6 or 22 or 23$)$ with $\tilde{l}_{K}=1$, decreasing $\tilde{l}_{K}$ increases the cut off value of $k$.

\section{fluid}

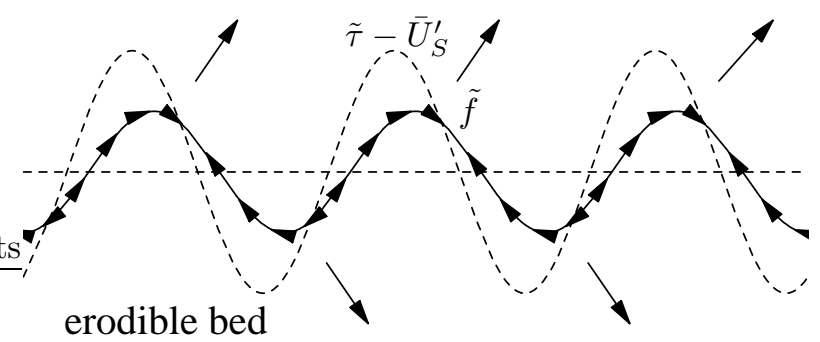

Figure 4: A wavy profile (bold line, $\tilde{f}$ ) has a perturbation of skin friction (dashed line, $\left.\tilde{\tau}-\bar{U}_{S}^{\prime}\right)$ in advance of phase. When it is positive, the matter is moved down stream (small arrows on the profile), when is is negative, it is in opposite direction. The result is an increase of the wave and a displacement in the stream direction (large inclined arrows).

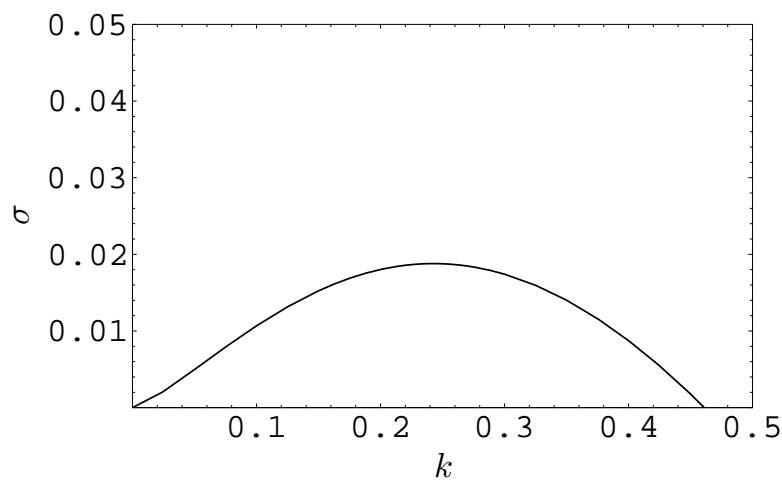

Figure 5: Amplification factor function of wave number. Averaged oscillating case, $\Lambda=1$ case ( 4 and 27 ). 


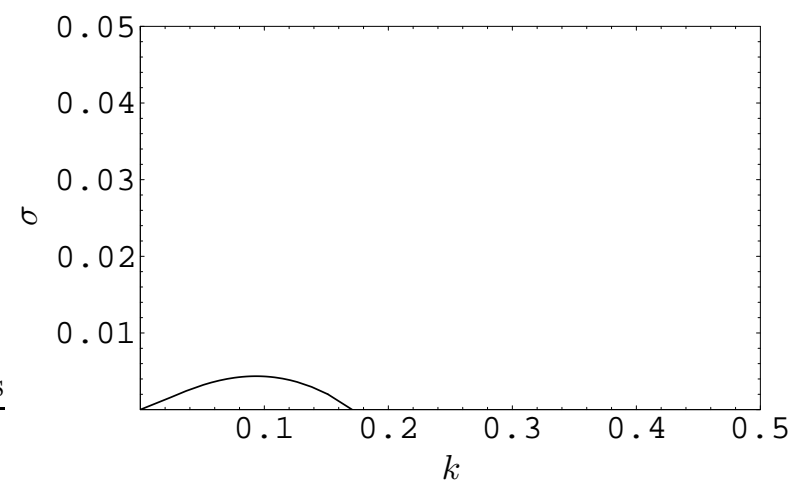

Figure 6: Amplification factor function of wave number. Averaged oscillating case, $\tilde{l}_{K}=1$ case $(6$ and 28$)$.

\section{fluid}

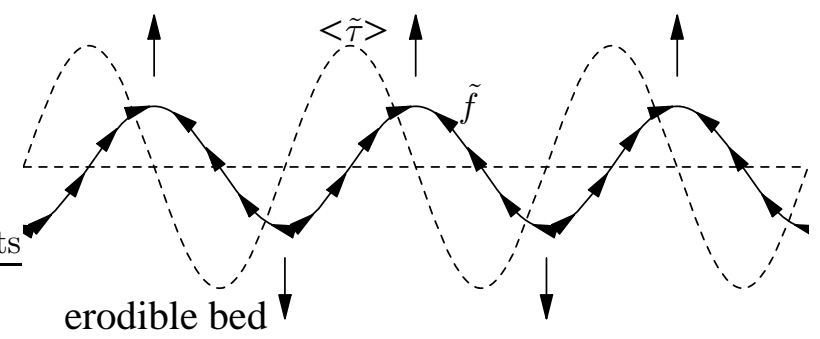

Figure 7: A wavy profile (bold line, $\tilde{f}$ ) has a mean perturbation of skin friction (dashed line, $<\tilde{\tau}>$ ) out of phase. When $\langle\tilde{\tau}>$ is positive, the matter is moved from left to right (small arrows on the profile), when it is negative, it is in opposite direction. The result is an increase of the wave without displacement (large vertical arrows).

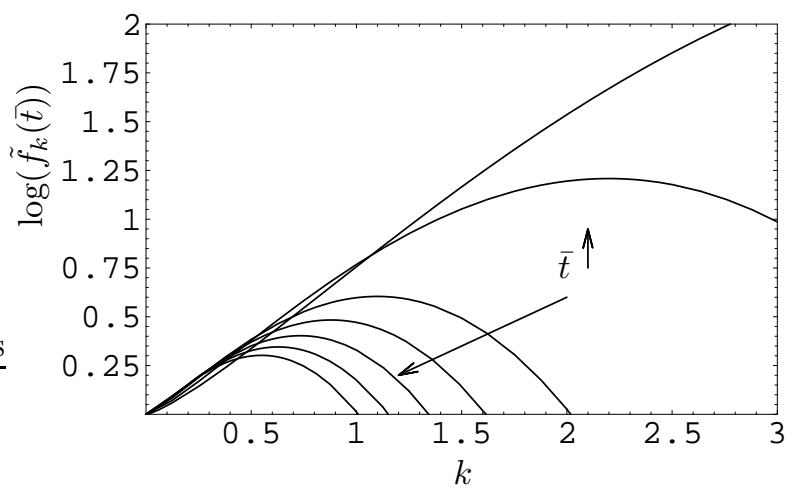

Figure 8: Decelerated case $\Lambda=1$ (21 and 30), plot of $\log \left(\tilde{f}_{k}(\bar{t})\right)$ as function of $k$ for various increasing times. As time increases, short waves are more and more stabilised. 


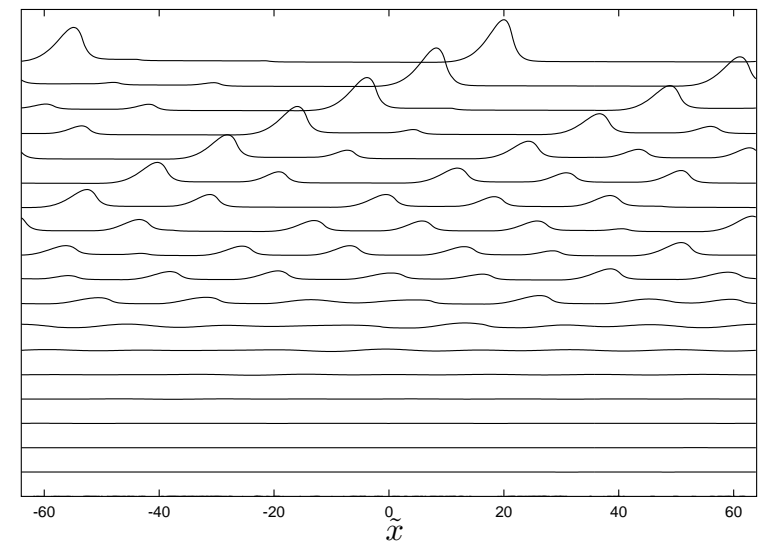

Figure 9: Steady case, with (22) with $\bar{U}_{S}^{\prime}=1, \tilde{l}_{s}=1$. Spatio-temporal diagram $(t=0,20,40, \ldots$ from bottom to top). The flow is from left to right. Starting from a random small noise, structures emerge and merge.

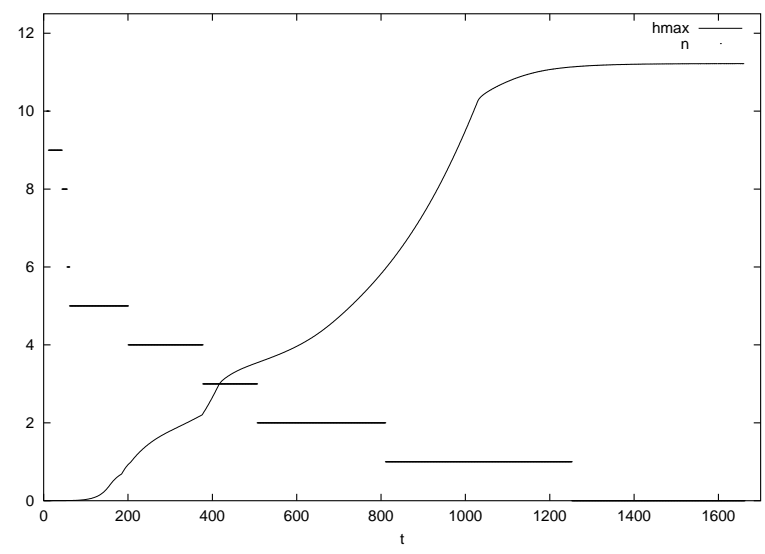

Figure 10: Steady case $\bar{U}_{S}^{\prime}=1, \tilde{l}_{s}=1$, evolution of the maximum value and of the number of bumps in the domain versus time. 


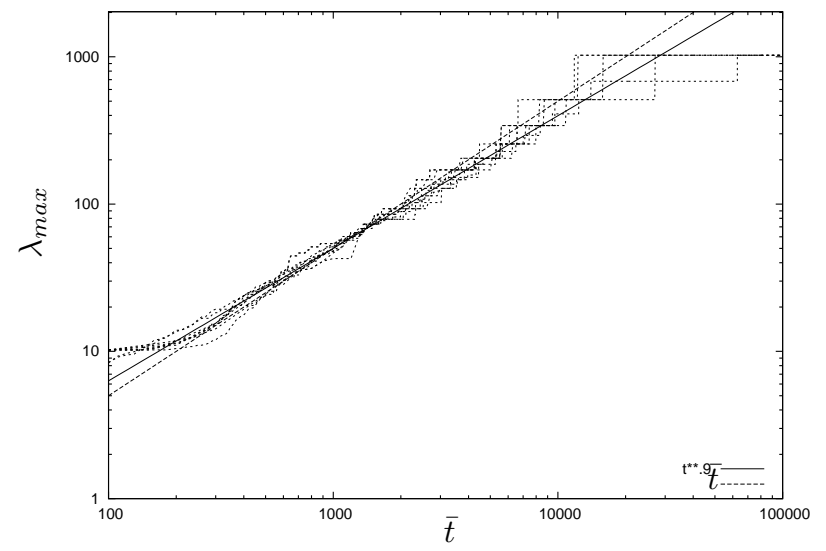

Figure 11: Constant shear, the wave length of the structure scales with a power between $\bar{t}^{0.9}$ and $\bar{t}$.

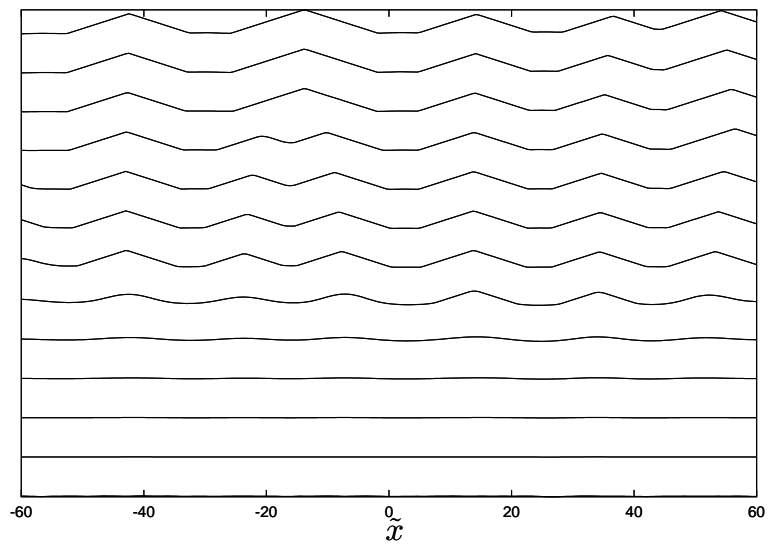

Figure 12: Oscillating régime with (22) and slope limitation $V=1, \frac{1}{\mu}=0.05$, spatio-temporal diagram, time increases from bottom to top 


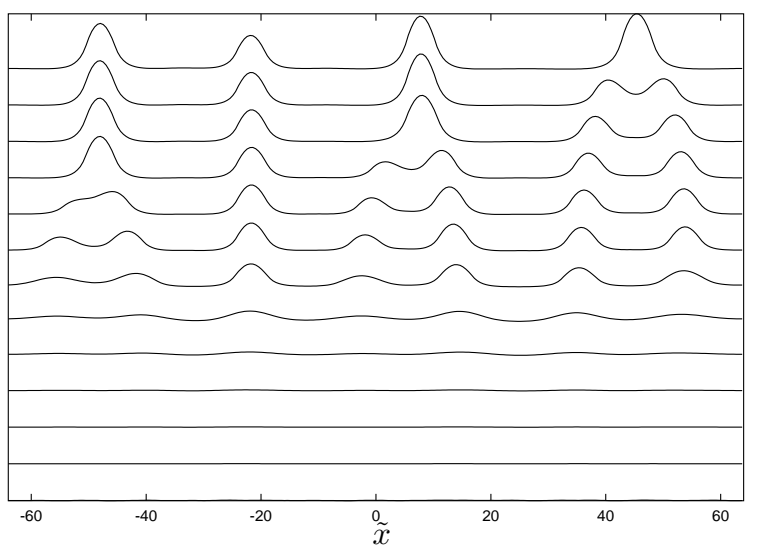

Figure 13: Oscillating régime with (22), spatio temporal diagram, time increases from bottom to top. Ripples growth from a random noise and merge two by two.

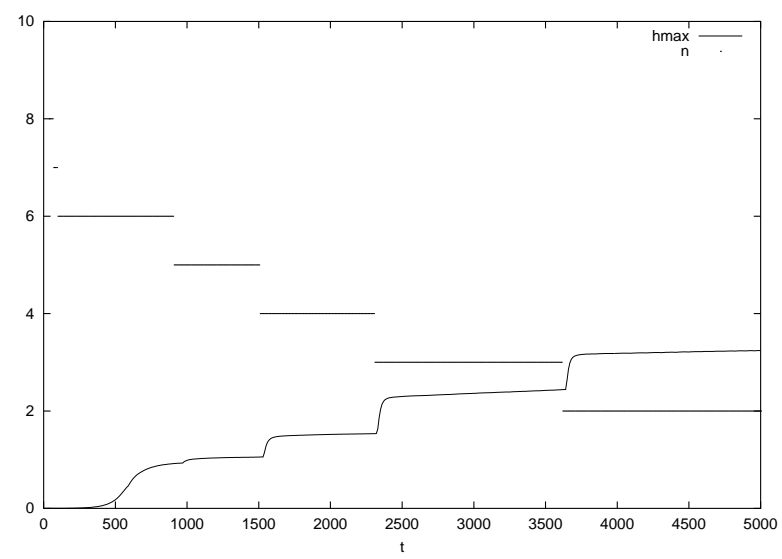

Figure 14: oscillating case $\bar{U}_{S}^{\prime}=\cos (\bar{t}), \tilde{l}_{K}=1$, evolution of the maximum height of the bumps, and number of bumps in the domain versus time. 


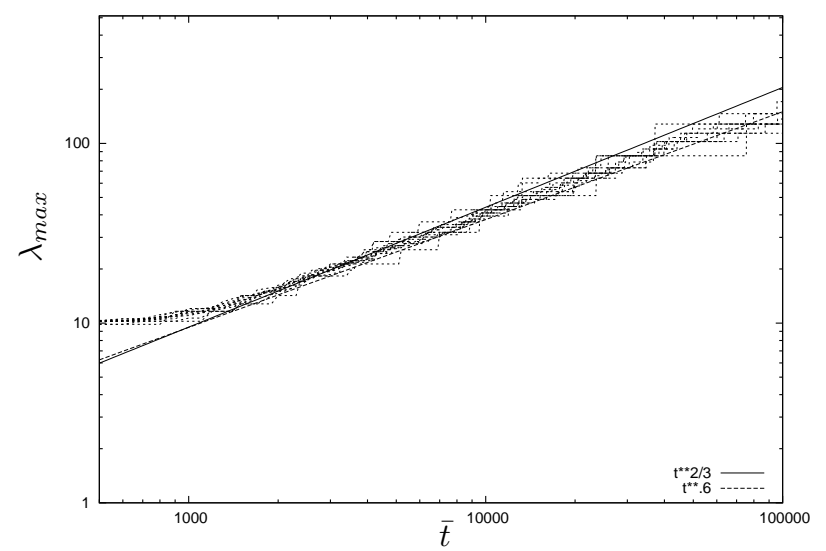

Figure 15: Oscillating shear, the wave length of the structure scales with a power law between $\bar{t}^{0.6}$ and $\bar{t}^{2 / 3}$.

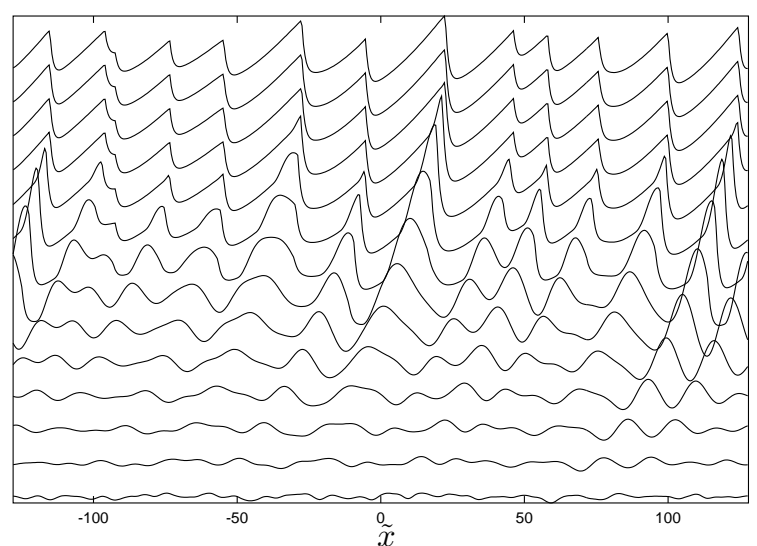

Figure 16: Decelerated case with $(23) l_{s}=1$. Spatio- temporal diagram, time increases from bottom to top. There is a final steady bed because the shear stress is under the threshold. 


\section{References}

[1] B. Andreotti, P. Claudin P. and S. Douady, "Selection of dune shapes and velocities. Part 2: A two-dimensional modelling", Eur. Phys. J., B 28, pp 341-352, (2002).

[2] R. I. Bowles \& F. T. Smith, "The standing jump: theory, computations and comparisons with experiments", J. F. M., vol. 242, pp. 145-168, (1992).

[3] A. J. Bray, Domain growth and coarsening, in Phase Transitions and Relaxation in Systems with Competing Energy Scales, edited by T. Riste and D. Sherrington, NATO ASI Series C, Vol.415, pp.405-436, Kluwer Academic, (1993).

[4] H. Caps, "Instabilités des interfaces fluide/ granulaire, études expérimentales", $\mathrm{PhD}$ thesis University of Liège.

[5] F. Charru, H. Mouilleron- Arnould, "Instability of a bed of particules sheared by a viscous flow", J. Fluid Mech., 452, pp. 303 - 323, (2002).

[6] NASA, Jet Propulsion Laboratory http://www.jpl.nasa.gov/releases/2004/90.cfm

[7] J. Fredsøe and R. Deigaard, "Mechanical of Coastal Sediment Transport", Advanced Series on Ocean Engineering - Vol. 3, World Scientific, 392 p (1992).

[8] Fowler, A.C. (2001): " Dunes and drumlins", In: Geomorphological fluid mechanics, eds. A. Provenzale and N. Balmforth, pp. 430-454, Springer-Verlag, Berlin.

[9] J. Gajjar \& F.T. Smith, "On hypersonic self induced separation, hydraulic jumps and boundary layer with algebraic growth", Mathematika, 30, pp. 77-93, (1983).

[10] K.K.J. Kouakou, H. Caps \& P.-Y. Lagrée (2003): " Comparaisons numériques, analytiques et asymptotiques pour l'écoulement sur une dune sous marine.", Congrès Franç ais de Mécanique; Nice 01-05 sept. 2003

[11] K. Kroy G. Sauerman \& J. Hermann (2002): "A minimal model for sand dunes", phys Rev Lett, vol 88, 05431.

[12] Lagrée P.-Y. (2000): "Erosion and sedimentation of a bump in fluvial flow", C. R. Acad. Sci. Paris, t328, Série II b, pp. 869-874.

[13] P.-Y. Lagrée (2003): "A Triple Deck model of ripple formation and evolution", Physics of Fluids, Vol 15 n 8, pp. 2355-2368

[14] V. Ya Neiland, "Propagation of perturbation upstream with interaction between a hypersonic flow and a boundary layer", Mekh. Zhid. Gaz. 4 pp 53-57, (1969).

[15] Peter Nielsen, "Coastal Bottom Boundary Layers and Sediment Transport", Advanced Series on Ocean Engineering - Vol. 4, World Scientific, 340p (1992).

[16] W. H. Press, B. P. Flannery, S. A. Teukolsky, and W. T. Vetterling, Numerical Recipes in C : The Art of Scientific Computing, Cambridge University Press (2002).

[17] G. Rousseaux, A. Stegner, J.E. Wesfreid (2004): "Wavelength selection of rolling grain ripples in laboratory",. Phys. Rev. E, 69, 031307, 2004 
[18] G. Rousseaux, H. Yoshikawa, A. Stegner, J.E. Wesfreid (2004): "Dynamics of transient eddy above rolling-grain ripples", Phys. Fluids, 16, pp. 1049-1058.

[19] G. Sauerman, K. Kroy \& J. Hermann (2001): "A continuum saltation model for sand dunes", phys Rev Lett, vol 64, 031305.

[20] M.A. Sherer, F. Melo, \& M. Marder (1999): " Sand ripple in an oscillating sand- water cell", Physics of Fluids, vol 11, n 1, pp. 58-67.

[21] F.T. Smith, "On the high Reynolds number theory of laminar flows", IMA J. Appl. Math. 28, pp 207-281, (1982).

[22] Smith FT, Brighton PW M., Jackson PS \& Hunt JCR "Onboundary layer flow past wo dimensional obstacles", JFM 110, pp 1-37 (1981).

[23] K. Stewartson \& P.G. Williams (1969) "Self-induced separation", Proc. Roy. Soc. A 312 pp. 181-206 (1969).

[24] H. Schlichting, "Boundary layer theory" 7th ed Mc Graw Hill, NewYork, 748p (1987).

[25] V. V. Sychev, A. I. Ruban, V. V. Sychev \& G. L. Korolev, " Asymptotic theory of separated flows", Cambridge U.P., 352 p, (1998).

[26] M. Van Dyke, "Perturbation Methods in Fluid Mechanics" Parabolic Press, Standford, 271p, (1975).

[27] S. Villain-Guillot, and C. Josserand, "Non-linear growth of periodic interface", Phys. Rev. E 66, 036308 (2002).

[28] C.T. Yang, "Sediment transport: theory \& practice", Mc Graw Hill Education Europe, 480p (1995). 